\title{
Mapping the circumstellar SiO maser emission in R Leonis
}

\author{
R. Soria-Ruiz ${ }^{1}$, J. Alcolea ${ }^{2}$, F. Colomer ${ }^{3}$, V. Bujarrabal ${ }^{3}$, and J.-F. Desmurs ${ }^{2}$ \\ 1 Joint Institute for VLBI in Europe, Postbus 2, 7990 AA Dwingeloo, The Netherlands \\ e-mail: soria@jive.nl \\ 2 Observatorio Astronómico Nacional, Alfonso XII 3, 28014 Madrid, Spain \\ 3 Observatorio Astronómico Nacional, Apartado 112, 28803 Alcalá de Henares, Spain
}

Received 27 March 2007 / Accepted 4 April 2007

ABSTRACT

Context. The study of the innermost circumstellar layers around AGB stars is crucial for understanding how these envelopes are formed and evolve. The SiO maser emission occurs at a few stellar radii from the central star, providing direct information on the stellar pulsation and on the chemical and physical properties of these regions. Our data also shed light on several aspects of the $\mathrm{SiO}$ maser-pumping theory that are not yet well understood.

Aims. We aim to determine the relative spatial distribution of the $43 \mathrm{GHz}$ and $86 \mathrm{GHz} \mathrm{SiO}$ maser lines in the oxygen-rich evolved star R Leo.

Methods. We imaged the $43 \mathrm{GHz}\left({ }^{28} \mathrm{SiO} v=1,2 J=1-0\right.$ and $\left.{ }^{29} \mathrm{SiO} v=0 J=1-0\right)$ and $86 \mathrm{GHz}\left({ }^{28} \mathrm{SiO} v=1 J=2-1\right.$ and ${ }^{29} \mathrm{SiO} v=0$ $J=2-1$ ) masing regions with milliarcsecond resolution, by means of very long baseline interferometry.

Results. We confirm previous results obtained in other oxygen-rich envelopes. In particular, when comparing the $43 \mathrm{GHz}$ emitting regions, the ${ }^{28} \mathrm{SiO} v=2 J=1-0$ transition is produced in an inner layer, slightly closer to the central star than the $v=1 J=1-0$. On the other hand, the $86 \mathrm{GHz}^{28} \mathrm{SiO} v=1 J=2-1$ line arises clearly in a farther shell. We have also mapped the ${ }^{29} \mathrm{SiO} v=0 J=$ $1-0$ emission in R Leo for the first time. The already reported discrepancy between the observed distributions of the different maser lines and the theoretical predictions is also found in R Leo.

Key words. radio lines: stars - masers - techniques: interferometric - stars: circumstellar matter - stars: AGB and post-AGB

\section{Introduction}

Along the asymptotic giant branch phase, many stars exhibit maser amplification in different molecular lines. In oxygen-rich stars $([\mathrm{O}] /[\mathrm{C}]>1)$, O-bearing compounds are mainly formed and maser emission is present in $\mathrm{SiO}, \mathrm{H}_{2} \mathrm{O}$, and $\mathrm{OH}$. The study of these different molecules provides information on the overall envelope, from the inner layers dominated by the stellar pulsation ( $\mathrm{SiO}$ masers) to the outermost regions where the circumstellar material is expanding at constant velocity ( $\mathrm{OH}$ masers).

The very long baseline interferometry is a unique technique for studying the compact and very bright $\mathrm{SiO}$ emission, so it is particularly helpful in understanding the different and complex processes occuring in these inner regions of the envelope. On the other hand, the current models of pumping, either collisional or radiative, do not reproduce some characteristics of the $\mathrm{SiO}$ masers that have been observed, such as their relative location in the envelope. To test these models and constrain the physical parameters of these inner shells better, it is very useful to perform simultaneous observations of several maser transitions. For this reason, we carried out multi-line and multi-epoch observations in a sample of AGB stars. We present the latest results for the Mira-type variable R Leo.

\section{VLBA observations}

The observations were done with the $\mathrm{NRAO}^{1}$ Very Long Baseline Array (VLBA) on 2002 December 7. Nearly

1 The National Radio Astronomy Observatory is a facility of the National Science Foundation operated under cooperative agreement by Associated Universities, Inc. simultaneous observations of different $43 \mathrm{GHz}$ and $86 \mathrm{GHz}$ ${ }^{28} \mathrm{SiO}$ and ${ }^{29} \mathrm{SiO}$ maser transitions were performed on the variable star R Leo. The $86 \mathrm{GHz}$ lines were observed in between the two $43 \mathrm{GHz}$ scans, and, therefore, we can assume for our purposes that the observations were simultaneous.

The data correlation was done at the VLBA correlator located in Socorro (New Mexico). Left and right circular polarizations (LCP \& RCP) were measured for the ${ }^{28} \mathrm{SiO} v=1$ and $v=2$ $J=1-0$ lines, whereas only the LCP was observed in the other transitions. Since no significant difference was found between the maps, less than 5\%, the final image is the average of both polarizations.

Standard procedures for spectral-line VLBI data reduction were followed in the calibration and production of the maps. The amplitude was calibrated using the system temperatures and antenna-gain corrections for the $86 \mathrm{GHz}$ and ${ }^{29} \mathrm{SiO}$ data and the template method for the other $43 \mathrm{GHz}$ data. The phase errors were removed in a two-step process: first, the single-band delay corrections were derived from the continuum calibrators, OJ287 and 3C 273. Second, the fringe-rates were estimated by selecting a bright and simple-structured channel, and the corrections found were subsequently applied to the maser source. The maps were produced using the CLEAN deconvolution algorithm.

\section{Results and data fits}

The results are presented as follows (Figs. 1 and 2). For each observed line, we show the integrated emission map in Jy beam ${ }^{-1} \mathrm{~km} \mathrm{~s}^{-1}$ units (center panel), the spectrum of the crosscorrelated emission for the different maser components (numbered panels), the total power spectrum (AC) of one of the 

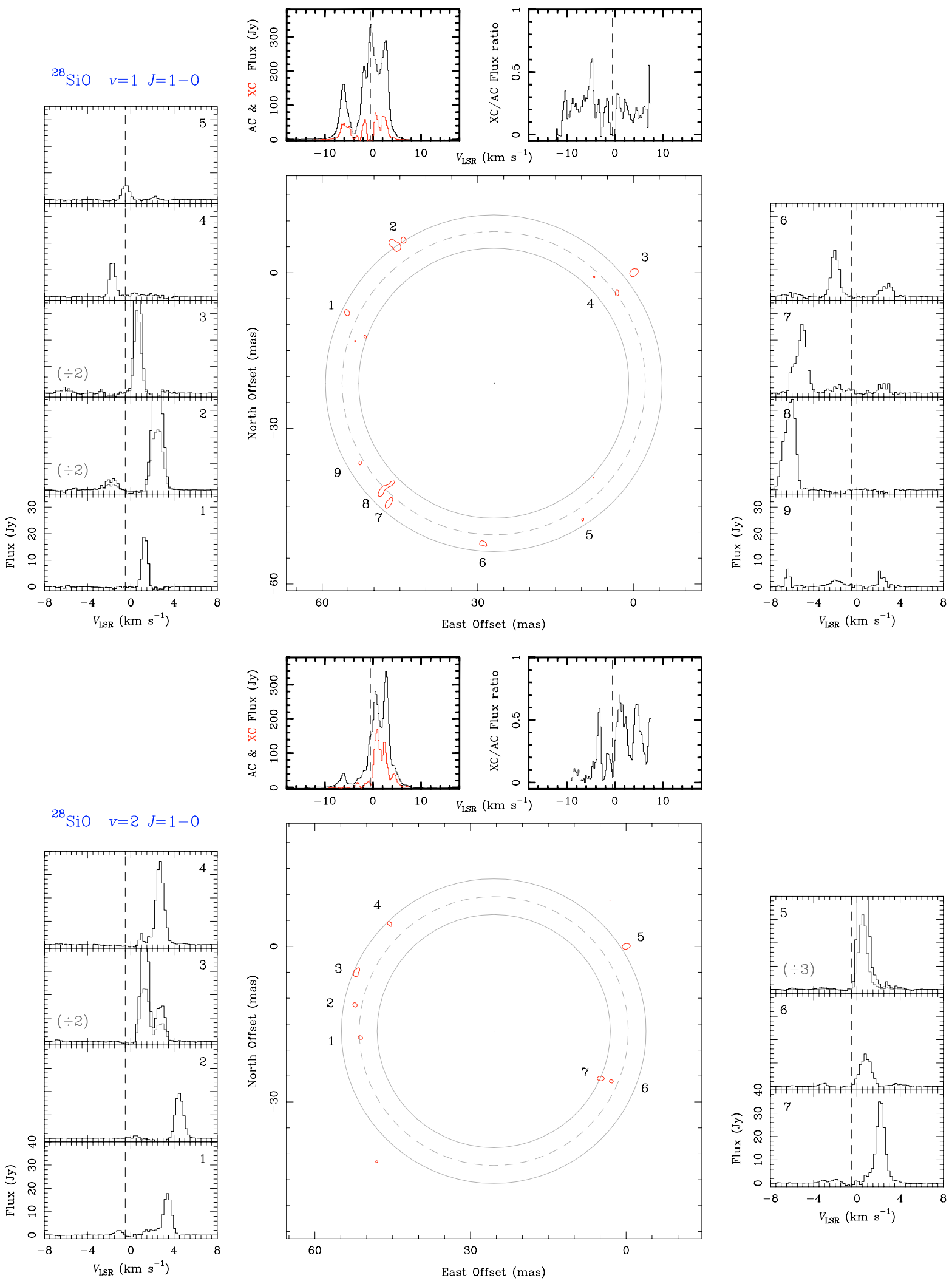

Fig. 1. The ${ }^{28} \mathrm{SiO} v=1$ (upper panel) and $v=2$ (lower panel) $J=1-0$ maser emission in R Leo. Each figure shows the integrated intensity map in Jy beam ${ }^{-1} \mathrm{~km} \mathrm{~s}^{-1}$ units, the spectra of the individual maser components, the total power spectrum and the emission in the map, and their ratio. For some maser components, the intensity has been divided by a factor of 2 or 3 to facilitate the comparison with the other spectra. The vertical dashed lines indicate the systemic velocity of the source, $V_{\mathrm{LSR}}=-0.5 \mathrm{~km} \mathrm{~s}^{-1}$ (Bujarrabal et al. 1989). Circles represent the fits for the masing regions (dashed: mean radius $\bar{R}$, continuous: $R_{\text {out }}$ and $R_{\text {in }}$ defined as $\bar{R} \pm \frac{1}{2} \triangle R$ ) (see Sect. 3). The peak intensity is $28.45 \mathrm{Jy} \mathrm{beam}^{-1} \mathrm{~km} \mathrm{~s}^{-1}(v=$ 1) and $53.5 \mathrm{Jy} \mathrm{beam}^{-1} \mathrm{~km} \mathrm{~s}^{-1}(v=2)$, and the shown contour is equivalent to the $5 \sigma$ level, with $\sigma=0.4 \mathrm{Jy} \mathrm{beam}^{-1} \mathrm{~km} \mathrm{~s}^{-1}(v=1)$ and $\sigma=$ $0.7 \mathrm{Jy}_{\text {beam }}^{-1} \mathrm{~km} \mathrm{~s}^{-1}(v=2)$. 

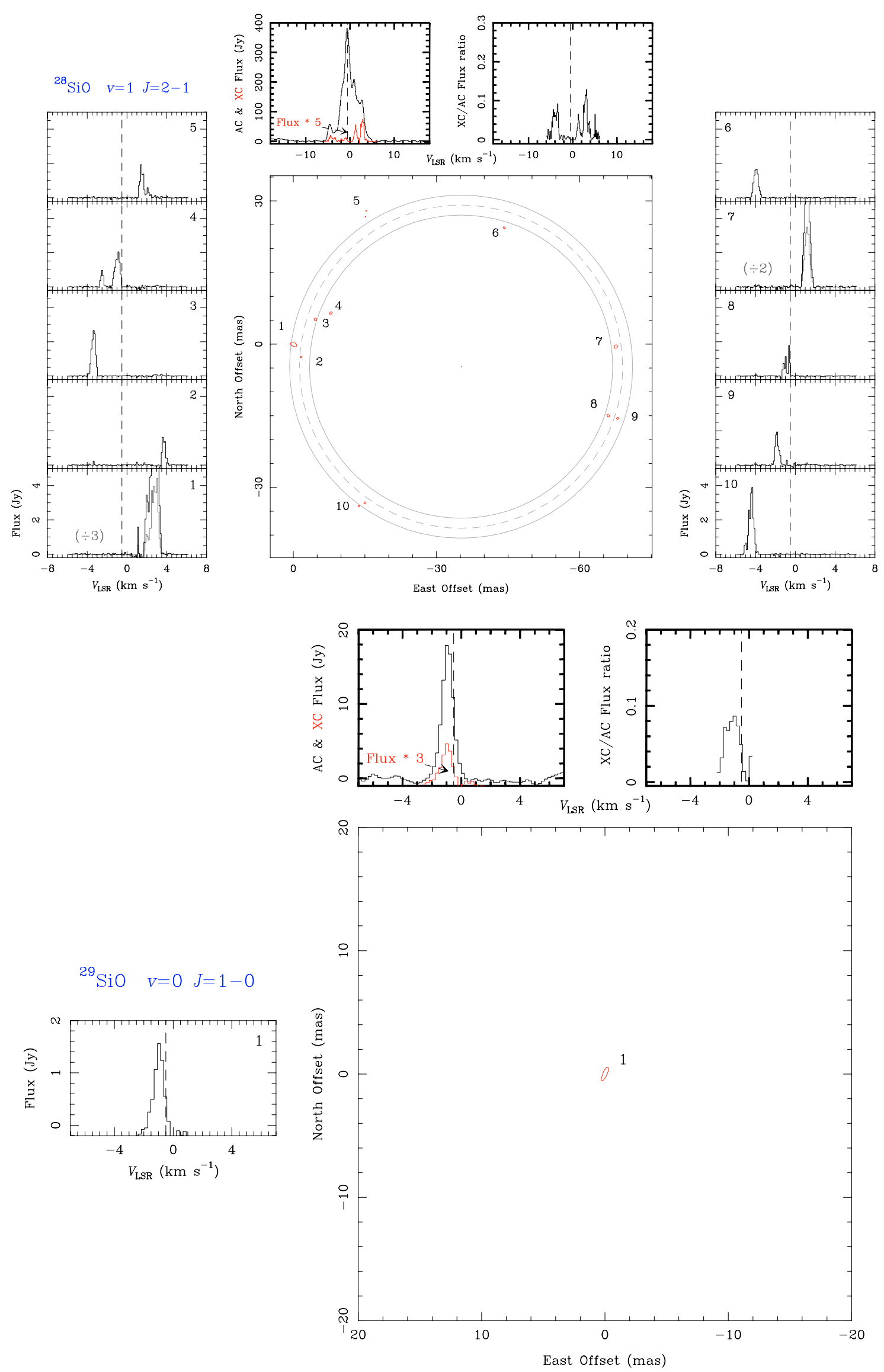

Fig. 2. Same as Fig. 1 for the ${ }^{28} \mathrm{SiO} v=1 J=2-1$ (upper panel) and ${ }^{29} \mathrm{SiO} v=0 \mathrm{~J}=1-0$ (lower panel) maser emission in R Leo. The peak intensity is $6.03 \mathrm{Jy}$ beam ${ }^{-1} \mathrm{~km} \mathrm{~s}^{-1}\left({ }^{28} \mathrm{SiO}\right)$ and $0.26 \mathrm{Jy}$ beam ${ }^{-1} \mathrm{~km} \mathrm{~s}^{-1}\left({ }^{29} \mathrm{SiO}\right)$, and the shown contour is equivalent to the $5 \sigma$ level, with $\sigma=$ $0.08 \mathrm{Jy}_{\text {beam }}{ }^{-1} \mathrm{~km} \mathrm{~s}^{-1}\left({ }^{28} \mathrm{SiO}\right)$ and $\sigma=0.01 \mathrm{Jy}_{\text {beam }}{ }^{-1} \mathrm{~km} \mathrm{~s}^{-1}\left({ }^{29} \mathrm{SiO}\right)$. 
Table 1. Observed maser transitions and results of the fits.

\begin{tabular}{|c|c|c|c|c|c|}
\hline Specie & nsition & $\begin{array}{c}\text { Restoring } \\
\left.\text { beam }\left(m^{2}\right)^{2}\right)\end{array}$ & $\begin{array}{c}\bar{R} \\
(\mathrm{mas})\end{array}$ & $\begin{array}{c}\triangle R \\
(\mathrm{mas})\end{array}$ & $\begin{array}{l}\text { Center } \\
\left(X_{\mathrm{c}}, Y_{\mathrm{c}}\right)\end{array}$ \\
\hline${ }^{28} \mathrm{SiO} v=1$ & $J=1-0$ & $0.78 \times 0.50$ & 29.24 & 6.42 & $(26.9,-21.3)$ \\
\hline$v=1$ & $J=2-1$ & $0.50 \times 0.50$ & 33.84 & 4.20 & $(-35.2,-4.7)$ \\
\hline$v=2$ & $J=1-0$ & $0.50 \times 0.50$ & 25.92 & 6.88 & $(25.5,-16.3)$ \\
\hline${ }^{29} \mathrm{SiO} v=0$ & $J=1-0$ & $0.78 \times 0.22$ & one spot & - & - \\
\hline$v=0$ & $J=2-1$ & non-det. & - & - & - \\
\hline
\end{tabular}

VLBA antennas and of the emission in the map (XC) (upperleft panel), and the ratio of these two magnitudes (upper-right panel). We also estimated the size of the total masing regions by fitting our data to rings. Only those components with $S N R \geq$ 6 have been included in the fits. The results derived from the calculations are summarized in Table 1: characteristic ring radius $(\bar{R})$, ring width $(\triangle R)$, and center of the ring $\left(X_{\mathrm{c}}, Y_{\mathrm{c}}\right)$ (see details on the fitting process in Soria-Ruiz et al. 2005). In particular, the angular sizes derived for the ${ }^{28} \mathrm{SiO} v=1$ and $v=2$ $J=1-0$ regions (Table 1 ) are compatible with previous observations performed in R Leo by Cotton et al. (2004). Among the six transitions observed, only the ${ }^{29} \mathrm{SiO} v=0 J=2-1$ has not been detected. A more detailed description of the maps is given in the next section.

\section{Relative spatial distribution and pumping mechanisms}

Our maps show that the spatial distribution of the $v=1$ and $v=$ 2 maser spots is similar, although not all the components appear in both transitions (Fig. 1). Concerning the relative location of the $43 \mathrm{GHz}{ }^{28} \mathrm{SiO}$ maser layers, the $v=2$ emission is produced in a closer region of the envelope, assuming that the centroids of all the emissions are coincident. This is also consistent with previously reported results in other oxygen-rich envelopes (see e.g. Desmurs et al. 2000; Cotton et al. 2004; Soria-Ruiz et al. 2004, 2005). In contrast to the $43 \mathrm{GHz}$ regions, this first map of the ${ }^{28} \mathrm{SiO} v=1 J=2-1$ emission in R Leo reveals that the components of this maser line are situated in a significant outer region of the envelope, with a very different spot distribution. Since the $J=2-1$ emission has only been imaged in a very few sources, this result for R Leo is particularly important for testing the proposed $\mathrm{SiO}$ maser mechanisms. Finally, the ${ }^{29} \mathrm{SiO} v=0$ $J=1-0$ map consisted of one maser spot, thus making it difficult to derive any spatial information. The total power and recovered emission are shown in Fig. 2.

Current pumping models, either radiative (Bujarrabal 1994a,b) or collisional (Humphreys et al. 2002), predict that the different rotational maser lines within the same vibrational state are produced under similar conditions and therefore are expected to be located in the same region of the envelope. As previously mentioned, we find a contradiction between these theoretical predictions and our observational results. This discrepancy has also been observed in other oxygen-rich stars; IRC +10011 (Soria-Ruiz et al. 2005) and TX Cam (Soria-Ruiz et al. 2006). Further calculations of the excitation of the $\mathrm{SiO}$ molecule in AGB stars have shown that the conditions under which the different maser transitions occur change drastically when the line overlap between infrared lines of $\mathrm{H}_{2} \mathrm{O}$ and ${ }^{28} \mathrm{SiO}$ is introduced into the pumping models (Bujarrabal et al. 1996; Soria-Ruiz et al. 2004); such a mechanism could explain the lack of coincidence between the spots of different $J$-transitions within a vibrational state.

Although these new maps support the relevance of line overlaps in the $\mathrm{SiO}$ maser pumping in O-rich shells, we nevertheless think that similar studies should be performed for a larger number of evolved stars. In particular, it would be necessary to have data on all types of long-period variable stars, namely, Mira-type, semirregular, and irregular variables, as well as supergiant stars.

Acknowledgements. This work has been financially supported by the Spanish DGI (MCYT) under projects AYA2000-0927 and AYA2003-7584. All plots were made using the GILDAS software package (http://wWw. iram. fr/IRAMFR/GILDAS).

\section{References}

Bujarrabal, V. 1994a, A\&A, 285, 953

Bujarrabal, V. 1994b, A\&A, 285, 971

Bujarrabal, V., Gómez-González, J., \& Planesas, P. 1989, A\&A, 219, 256

Bujarrabal, V., Alcolea, J., Sánchez Contreras, C., \& Colomer, F. 1996, A\&A, 314,883

Cotton, W. D., Mennesson, B., Diamond, P. J., et al. 2004, A\&A, 414, 275

Desmurs, J.-F., Bujarrabal, V., Colomer, F., \& Alcolea, J. 2000, A\&A, 360, 189

Humphreys, E. M. L., Gray, M. D., Yates, J. A., et al. 2002, A\&A, 386, 256

Soria-Ruiz, R., Alcolea, J., Colomer, F., et al. 2004, A\&A, 426, 131

Soria-Ruiz, R., Colomer, F., Alcolea, J., et al. 2005, A\&A, 432, L39

Soria-Ruiz, R., Colomer, F., Alcolea, J., et al. 2006, Proceedings of the 8th EVN Symposium 